\title{
Novel Collective Thomson scattering system for determination of ion properties in a high flux plasma beam
}

\author{
H. J. van der Meiden ${ }^{1}$, J. W. M. Vernimmen ${ }^{1}$, K. Bystrov ${ }^{1}$, K. Jesko ${ }^{1,2}$, M. Y. Kantor ${ }^{3,5}$, G. De
} Temmerman $^{4}$ and T. W. Morgan ${ }^{1}$

${ }^{1}$ FOM Institute DIFFER, Dutch Institute for Fundamental Energy Research, Association EURATOM-FOM, Trilateral Euregio Cluster, P.O. Box 6336, 5600 HH Eindhoven, The Netherlands

${ }^{2}$ CEA, IRFM, F-13108 Saint-Paul-Lez-Durance, France

${ }^{3}$ loffe Institute, Russian Academy of Science, 194021 Saint Petersburg, Russia

4ITER Organization, Route de Vinon-sur-Verdon - CS 90046 - 13067 St Paul Lez Durance Cedex, France

${ }^{5}$ Association Euratom-Max-Planck-Institut für Plasmaphysik, D-85748 Garching bei Muenchen, Germany

\begin{abstract}
A Collective Thomson scattering system has been developed for measuring ion temperature, plasma velocity and impurity concentration in the high density magnetized Magnum-PSI plasma beam, allowing for measurements at low temperature $(<5 \mathrm{eV})$ and high electron density $>4 \times 10^{20} \mathrm{~m}^{-3}$, while avoiding laser plasma heating caused by inverse Bremsstrahlung. The Collective Thomson scattering system is based on the fundamental mode of a seeded Nd:YAG laser and equipped with a LIVAR M506 camera (EBABS technology). The first Collective Thomson scattering measurements are taken at the linear plasma generator Pilot-PSI, $40 \mathrm{~mm}$ downstream of the cascaded arc source. At this location the ion temperature is about equal to the electron temperature in the bulk of the plasma beam.
\end{abstract}

\section{Introduction}


In ITER, the heat flux to divertor plates has to be mitigated to be compatible with the power exhaust capability of the actively-cooled components, and operation in the so-called partially detached regime is required [1]. This regime of operation has been demonstrated in many tokamak experiments around the world and it has been found that the detached plasma state can be associated with particle recycling and ion-neutral friction in the near surface plasma, leading to cooling and temperature gradients along the magnetic field lines [1]. The requirements for power dissipation will be even more stringent in a DEMO reactor[2]. However, numerical models fail to reproduce the experimental observations in terms of rollover of the ion saturation current $[3,4]$ and the numerical simulations critically depend on high quality data to constrain the parameter space. Such high quality experimental data of the electron and ion population is presently lacking.

The advanced linear plasma generators Magnum-PSI $[5,6]$ and Pilot-PSI $[7,8]$ have the ability to produce ITER-relevant divertor plasma conditions with high density (electron density $>4 \times 10^{21} \mathrm{~m}^{-3}$ ) and low electron temperature $(<5 \mathrm{eV})$ both in steady-state and pulsed conditions [9]. This report demonstrates, that using novel state of the art diagnostics can fill this gap on these linear plasma generators.

First results obtained with a novel Collective Thomson scattering system (CTS), developed for Magnum-PSI, and tested at Pilot-PSI (Fig. 1) are presented. A combination of TS $[10,11]$ and CTS enables the simultaneous measurement of electron temperature $\left(T_{\mathrm{e}}\right)$, electron density $\left(n_{\mathrm{e}}\right)$, ion temperature $\left(T_{\mathrm{i}}\right)$ and plasma velocity ( $v_{\text {plasma }}$ ) with high accuracy (currently $25 \%$ at Pilot-PSI, but $<15 \%$ for Magnum-PSI system).

For CTS, the dimensions of the observed scattered wave, characterised by differential wave vector $\boldsymbol{k}$ must be comparable to or larger than the Debye length $\lambda_{\text {Debye }}[12,13]$. This relation is described by the so-called scattering parameter $\alpha$ defined as $\alpha=1 /|\boldsymbol{k}| \lambda_{\text {Debye. }}$. Here the size of the differential wave vector $k$ is equal to $k=|\boldsymbol{k}|=\left(4 \pi / \lambda_{0}\right) \sin (\theta / 2)$ with $\lambda_{0}$ the laser wavelength and $\theta$ the scattering angle between incident wave $\left(\boldsymbol{k}_{0}\right)$ and scattered wave $\left(\boldsymbol{k}_{\mathrm{s}}\right)$ (see insert in Fig. 1). 
Because it observes the collective rather than individual motion of the electrons, CTS enables observation of slow fluctuations like the velocity distribution of the ions and their temperature. In addition, CTS can distinguish the mass of the different ion species of the plasma as the Doppler broadening is inversely proportional to the square root of the ion mass (for a given $T_{\mathrm{i}}$ ), thus enabling direct measurement of seeding gas and impurity concentration. When $\alpha<<1$, the scattering process is referred to as incoherent TS and the individual electrons are scattered so that only the much broader spectrum of the so-called electron feature is observed, allowing for determination of $T_{\mathrm{e}}$ and $n_{\mathrm{e}}$.

This combination of scattering diagnostics will allow an accurate determination of the correlation between the plasma parameters close to the surface (1.5-20 mm away) and at the surface $\left(I_{\text {sat, }}\right.$, surface potential/temperature) and give key parameters for the processes occurring in the magnetic pre-sheath and electrostatic sheath [14]. Moreover, the $T_{i}$ and $v_{\text {plasma }}$ values obtained with CTS can be compared with those obtained with optical emission spectroscopy, this can be used for validating the coupling between ions and exited neutrals $H(n>2)[15]$.

\section{Theory}

As CTS effectively measures the Doppler broadening and shift of the Debye cloud, the measurement is difficult at low $T_{\mathrm{e}}$ and high ion mass $\left(m_{\mathrm{i}}\right)$. In this paper we show that CTS can be used in a high density (> $\left.4 \times 10^{20} \mathrm{~m}^{-3}\right)$, low temperature $(<5 \mathrm{eV})$ plasma beam for measurements of $T_{\mathrm{i}}$ and $v_{\text {plasma }}$ [16]. The scale difference in velocity between ions and electrons is large, the Salpeter approximation $[12,13]$ can be applied to describe the TS form factor $S(k, \omega)$ of the observed scattering spectrum as the sum of two separate contributions:

$$
S(k, \omega) d \omega \cong \frac{1}{\sqrt{\pi}} \Gamma_{\alpha}\left(x_{e}\right) d x_{e}+Z\left(\frac{\alpha^{2}}{1+\alpha^{2}}\right)^{2} \frac{1}{\sqrt{\pi}} \Gamma_{\beta}\left(x_{i}\right) d x_{i}
$$

with $x_{\mathrm{e}, \mathrm{i}}=\Delta \omega / \omega_{\mathrm{e}, \mathrm{i}}=\Delta \omega / k v_{\mathrm{e}, \mathrm{i}}$ the normalized wavelength with $v_{\mathrm{e}, \mathrm{i}}=\operatorname{sqrt}\left(2 k_{\mathrm{B}} T_{\mathrm{e}, \mathrm{i}} / m_{\mathrm{e}, \mathrm{i}}\right)$ the average thermal speed of the particles ( $m_{\mathrm{e}, \mathrm{i}}$ represents the electron and ion mass): 


$$
\Gamma_{\alpha}\left(x_{e}\right)=\frac{\exp \left(-x_{e}^{2}\right)}{\left|1+\alpha^{2} w\left(x_{e}\right)\right|^{2}} \text { and } \Gamma_{\beta}\left(x_{i}\right)=\frac{\exp \left(-x_{i}^{2}\right)}{\left|1+\beta^{2} w\left(x_{i}\right)\right|^{2}} \text { with } \beta=\sqrt{Z\left(\frac{\alpha^{2}}{1+\alpha^{2}}\right) \frac{T_{e}}{T_{i}}}
$$

where $Z$ is the effective charge of the ions, the values are obtained from [17] and are in the range of 11.2 for these low temperature plasmas. The plasma dispersion function $w\left(x_{\mathrm{e}, \mathrm{i}}\right)$, is tabulated by [18] and is described by a real $R w\left(x_{e, i}\right)$ and the Landau damping term $/ w\left(x_{e, i}\right)$ :

$$
\begin{aligned}
& R w\left(x_{e, i}\right)=1-2 x_{e, i} \exp \left(-x_{e, i}^{2}\right) \int_{0}^{x_{e, i}} \exp \left(p^{2}\right) d p \\
& I w\left(x_{e, i}\right)=-\sqrt{\pi} x_{e, i} \exp \left(-x_{e, i}^{2}\right)
\end{aligned}
$$

The first term of Eq. 1 describes the electron feature $\left(S_{e}\right)$ : if $\alpha<<1$, the spectrum will exhibit a Gaussian shape for a Maxwellian electron velocity distribution. $T_{\mathrm{e}}$ and $n_{\mathrm{e}}$ can be obtained from the width and the integral, respectively.

If $\alpha$ is well above 0.8 the contribution of the first term (Eq. 1) decreases and that of second term becomes significant, i.e. scattering from the electrons bunched in the Debye sphere of the ions, the socalled ion feature $\left(S_{\mathrm{i}}\right)$, becomes observable. If $T_{\mathrm{e}}$ is significantly smaller than $T_{\mathrm{i}}$ and thus $\beta<1$, the spectrum will resemble a Gaussian shape for a Maxwellian ion velocity distribution, i.e. $T_{\mathrm{i}}$ can be obtained from the spectral shape (see Fig. 2 and Eq. 5).

$$
S_{i}(k, \omega) d \omega=\frac{1}{\sqrt{\pi}} Z\left(\frac{\alpha^{2}}{1+\alpha^{2}}\right)^{2} \frac{\exp \left(-x_{i}^{2}\right)}{\left(1+\beta^{2} R w\left(x_{i}\right)\right)^{2}+\left(\beta^{2} I w\left(x_{i}\right)\right)^{2}} d x_{i}
$$

The dynamics of $\Gamma_{\beta}$ (Eq. 2) can be demonstrated by assuming that $\alpha \sim 0.8, Z=1$ and $T_{\mathrm{e}} \approx T_{\mathrm{i}}$. Then $\beta^{\sim} 0.6$, meaning that the shape of the ion feature spectrum (close to $x_{i}=0$ ) is mainly determined by the ratio of the exponential function and the real part of the plasma dispersion function in the denominator of $\Gamma_{\beta}\left(x_{i}\right)$, where the real part is highest at $x_{i}=0$, resulting in a flattened Gaussian (see Fig. 2) [19, 20, 21]. For $T_{\mathrm{e}}>T_{\mathrm{i}}$ ion acoustic resonances become dominant. Macroscopic velocities of the plasma can be determined from the Doppler shift of the spectrum of the ion feature [19]. The zero Doppler shift reference can be obtained from Rayleigh scattering. 
The region where $\alpha>0.8$ (typical operational range for this CTS system $n_{\mathrm{e}}^{\sim} 0.4-1 \times 10^{21} \mathrm{~m}^{-3}$ and $T_{\mathrm{e}}$ $\sim 1.4-3 \mathrm{eV}$, but in general terms $\left.n_{\mathrm{e}}\left(10^{21} \mathrm{~m}^{-3}\right)>0.3 T_{\mathrm{e}}(\mathrm{eV})\right)$, allows for a significant contribution of signal in the ion feature $S_{\text {i. }}$. If $\alpha=0.8$, this signal is $>11 \%$ of that of the electron feature (if $\alpha=0$ ) and for $\alpha=2$ this signal in $S_{i}$ is $36 \%$.

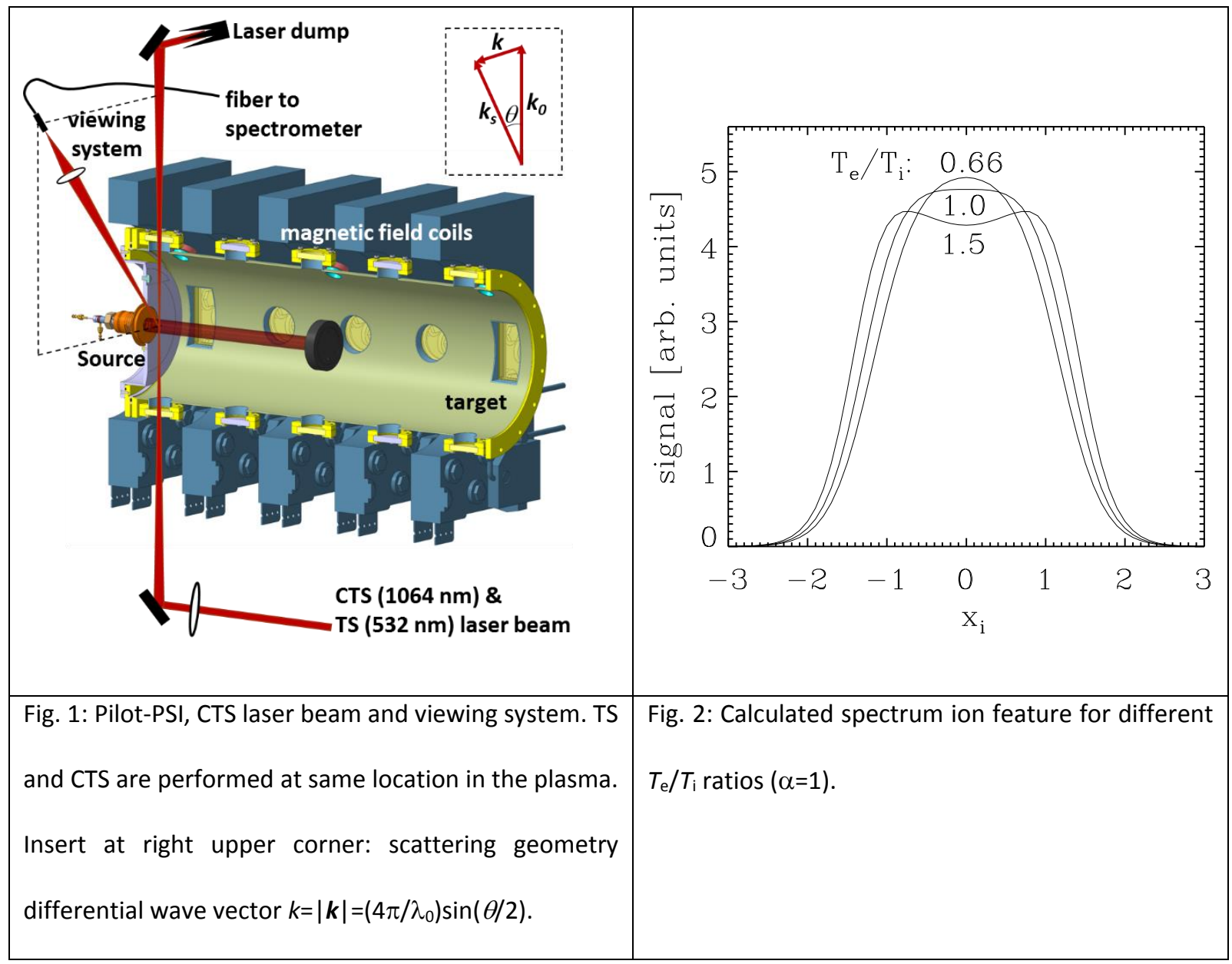

\section{Experimental Details}

The Pilot-PSI device is schematically shown in Fig. 1, and consists in a wall-stabilized DC cascaded arc which produces high flux plasma beams consisting of hydrogen $(H)$, deuterium (D), helium (He), neon (Ne), argon (Ar) or mixtures of these gases. The typical plasma parameters are a discharge current of $200 \mathrm{~A}$, gas flow of 3 standard litre per minute $\left(\mathrm{s} / \mathrm{m} ; 1 \mathrm{slm}=4.5 \times 10^{20}\right.$ particles $\left./ \mathrm{s}\right)$ and an axial magnetic field of $1.2 \mathrm{~T}$ to confine the plasma which interacts with a tungsten target located $560 \mathrm{~mm}$ downstream the source. 
The CTS laser system consists of a $10 \mathrm{~Hz}$ seeded laser (Continuum DLS 8000: pulse width of $7 \mathrm{~ns}$ FWHM, $1 \mathrm{~J} /$ pulse and linewidth $0.3 \mathrm{pm}$ at $1064 \mathrm{~nm}$ ). The location of the CTS scattering volume (beam waist $\sim 0.8 \mathrm{~mm}$ ) coincides with that of the incoherent TS system $\left(532 \mathrm{~nm}\right.$, scattering angle $\left.90^{\circ}\right)$. This wavelength (1064 $\mathrm{nm}$ ) is chosen to maximize $\alpha$ while still permitting the use of optical detection systems with excellent signal/noise specifications. The wavelength is also chosen such that laser plasma heating due to inverse Bremsstrahlung (proportional to wavelength to the second power [22]) is negligible.

The CTS $(1064 \mathrm{~nm})$ laser beam is coupled into the incoherent laser beam line by using a dichroic mirror (transmission for $532 \mathrm{~nm}$ and $90^{\circ}$ reflection for $1064 \mathrm{~nm}$ ), allowing for simultaneous TS and CTS measurements. The choice of different scattering angles and laser wavelengths of both systems is such that the $\alpha$ value for TS (fit function includes collective effects) is 5.4 times lower than that for CTS.

The detection branch comprises a viewing system (effective solid angle $1.9 \times 10^{-3} \mathrm{sr}$ ) that collects the Doppler broadened light at a scattering angle of $30^{\circ}$ (See Fig. 1, position scattering volume $40 \mathrm{~mm}$ downstream plasma source), a fiber bundle that relays the light to a spectrometer (spectral range $1.9 \mathrm{~nm}$, spectral resolution $0.0165 \mathrm{~nm}$ ) equipped with an Echelle grating operating at the blaze angle. The 2D camera is a LIVAR M506 EB-camera (EBABS technology, Electron Bombarded Active Pixel Sensor). The effective quantum efficiency is $\sim 25 \%$ at $1064 \mathrm{~nm}$ and exhibits an excellent $\mathrm{S} / \mathrm{N}$ ratio by virtue of the high EB gain. In order to suppress background light a 200 ns detection gate window was used.

The alignment position of the scattering volume (chord length $2 \mathrm{~mm}$ ) exhibited a radial uncertainty of 1-2 $\mathrm{mm}$ relative to the plasma center. This implies that for positions above and below the plasma center, artificially, reversed rotational velocities are observed.

\section{Results}

The data was fitted according to the ion feature equation (Eq. 5) (using an analytical approximation for the real part of the plasma dispersion function [23]), but convolved with the instrument 
function of the spectrometer. As fitting parameters $T_{\mathrm{i}}, \beta$, the Doppler shift and signal amplitude were used. $T_{\mathrm{e}}$ and $n_{\mathrm{e}}$ (error bar $8 \%$ and $4 \%$, respectively) and therefore $\alpha$ were measured independently and simultaneously by the incoherent TS system.
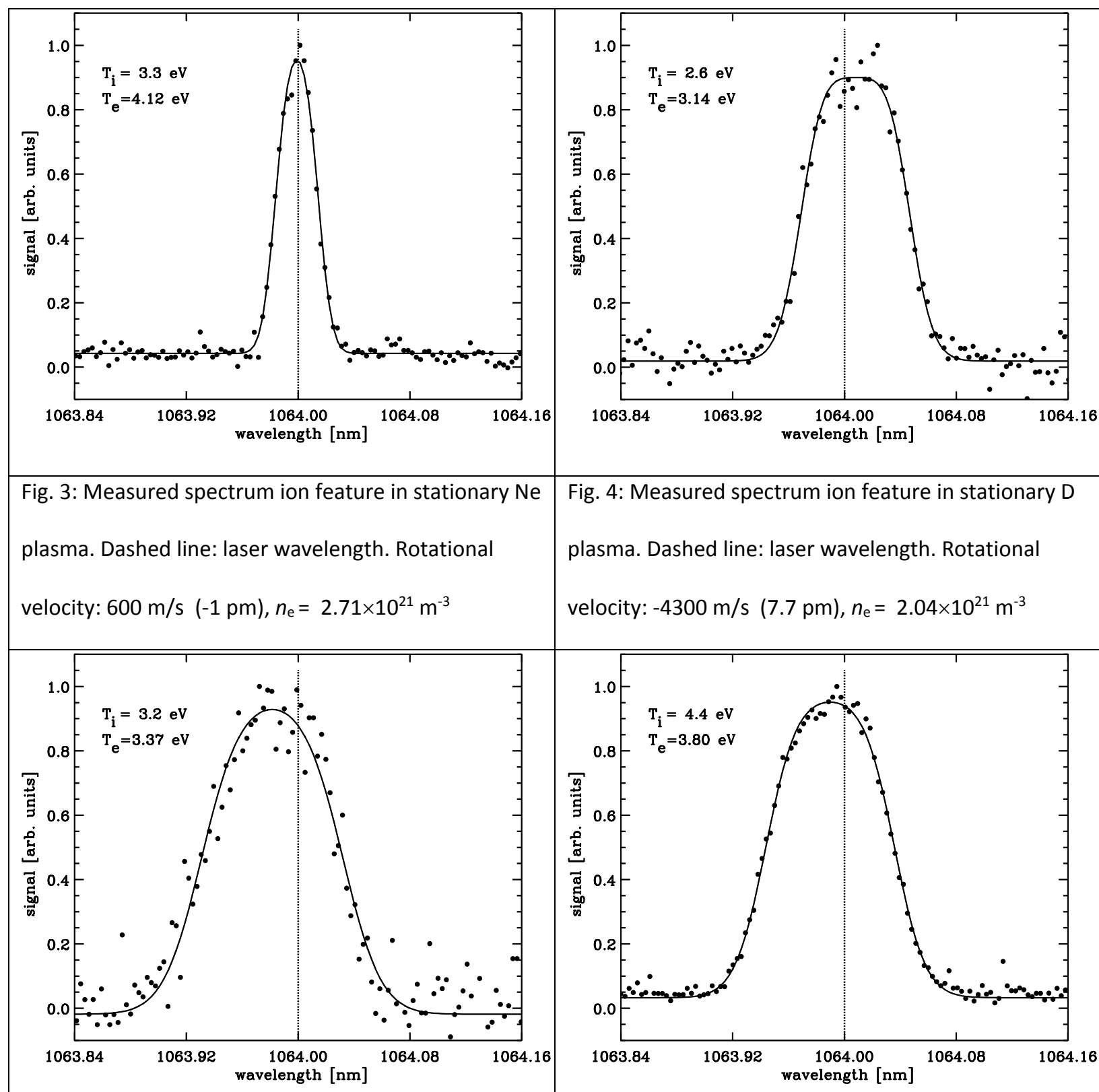

Fig. 4: Measured spectrum ion feature in stationary D plasma. Dashed line: laser wavelength. Rotational velocity: $-4300 \mathrm{~m} / \mathrm{s}(7.7 \mathrm{pm}), n_{\mathrm{e}}=2.04 \times 10^{21} \mathrm{~m}^{-3}$

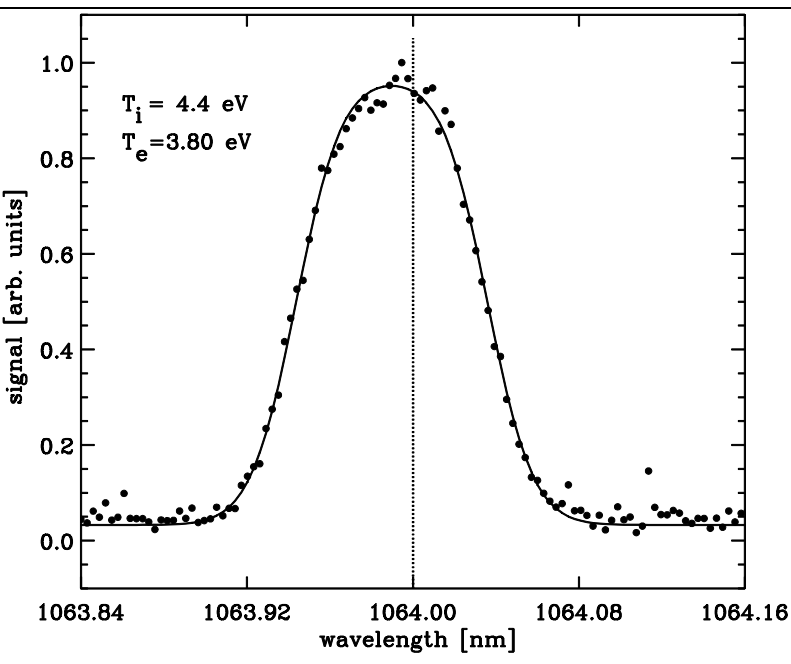

Fig. 5: Measured spectrum ion feature in stationary $\mathrm{H}$

Fig. 6: Measured spectrum ion feature in pulsed D plasma. Dashed line: laser wavelength. Rotational plasma. Dashed line: laser wavelength. Rotational velocity: $10400 \mathrm{~m} / \mathrm{s}(-18.2 \mathrm{pm}), n_{\mathrm{e}}=1.06 \times 10^{21} \mathrm{~m}^{-3}$ velocity: $5800 \mathrm{~m} / \mathrm{s}(-10.3 \mathrm{pm}), n_{\mathrm{e}}=6.2 \times 10^{21} \mathrm{~m}^{-3}$ 
In Fig. 3-5 ion feature spectra are shown corresponding to CTS on stationary plasmas. The signal was obtained by averaging CTS signal from 50 laser pulses ( $5 \mathrm{~s})$. The vertical dashed line in the figures represents the wavelength position of the laser (zero Doppler shift), measured by Rayleigh scattering. In Fig. 6 results are shown of CTS performed on a pulsed plasma; the high signal level is due to the high $n_{\mathrm{e}}$ of $6.2 \times 10^{21} \mathrm{~m}^{-3}$. The electron feature signal cannot be observed, because this spectrum will be smeared out, this spectrum is $\sim 43$ times $\left(\sqrt{m_{i} / m_{e}}\right)$ wider relative to that of hydrogen.

The spatial resolution of the system was $2 \mathrm{~mm}$ and due to this large sample length, rotational velocity smoothing effects are to be expected; the plasma exhibits steep rotational $v_{\text {plasma }}$ gradients [15] giving an indicative smoothing effect of $\sim 4000 \mathrm{~m} / \mathrm{s}$ for hydrogen, i.e. $\sim 7 \mathrm{pm}$.

The measured velocities indicated in Fig. 4-6 are significant, which means that the scattered signal is observed a few millimeter off-axis of the plasma (in the center the rotational $v_{\text {plasma }}$ is negligible). The accuracy of velocity determination, the shift is remarkably well visible by eye, is better than $10 \%$ for $v_{\text {plasma }}>4000 \mathrm{~m} / \mathrm{s}$, although it is here an averaged value. For $T_{i}$ determination the accuracy is determined by Poisson statistics, but in this case also by the mentioned velocity smoothing effect. For the measurements shown in Fig. 4-6 $T_{\mathrm{i}}$ was determined with $25 \%$ accuracy.

The width of the spectrum for Ne (Fig. 3) was close to that of the spectrometer instrument function, the error bar is here $>50 \%$. In Fig. 4 the shape of the fitted curve of the ion feature of the D plasma resembles closely the calculated curve, for $T_{\mathrm{e}}^{\sim} T_{\mathrm{i}}$ shown in Fig. 2; despite the influence of the instrument function the flattening in the center is discernible. Fig. 4-6 show that the ion and electron temperatures deviate maximally $18 \%$ for $\mathrm{D}$ and $\mathrm{H}$ plasma. Comparison of Fig. 3-5 shows clearly that the width of the ion feature spectra is dominated by the mass of the species of the plasma, demonstrating the ability to distinguish between different species in plasma mixtures [21]. The spectra show some fluctuations caused by the stray light fluctuations. 
The measured CTS velocities and ion temperatures are comparable with those determined by optical emission spectroscopy (OES) of the $H_{\beta}$ radiation [15]. Nevertheless, simultaneous measurements with OES and CTS in the near surface plasma and comparison of the results will be one of the tasks for the future.

\section{Conclusions and outlook}

In summary, simultaneous measurements of $T_{\mathrm{i}}, T_{\mathrm{e}}, n_{e}$ and the rotational plasma velocity have been demonstrated by a combination of an incoherent TS and a novel CTS system. In addition it has been shown that the different masses of the plasma species can be distinguished. For the used plasma conditions it was found that $T_{\mathrm{i}}$ is about equal to $T_{\mathrm{e}}$ at $40 \mathrm{~mm}$ downstream the nozzle of the source. The LIVAR M506 camera was deployed for scientific purposes. It is expected that this application will unleash new developments for TS. The CTS system is ready to be deployed in Magnum-PSI for the study of plasma properties close to a plasma-exposed surface, where different $T_{\mathrm{e}} / T_{\mathrm{i}}$ ratios are to be expected, due to sheath effects [27] and/or strong particle recycling processes. Moreover, the CTS system will be equipped with a multi-pass system $[24,25]$ ) enhancing the sensitivity by a factor 15 . An enhancement of the spectral resolution of a factor 2 will be achieved by having the grating of the spectrometer in double pass configuration. Because the system will be configured such that the CTS scattering plane is parallel to the magnetic field, only velocity components parallel to the magnetic field will be measured where only gentle axial velocity gradients are present. These upgrades will lead to accuracies better than $15 \%$. Finally it can be noted that this diagnostic is in principle a good measurement technique for measuring $T_{\mathrm{i}}$ and $v_{\text {plasma }}$ in the ITER divertor plasma [26]; up to date there is no good technique available for this task.

\section{Acknowledgements}

This work is part of the research programme of the Foundation for Fundamental Research on Matter (FOM), which is part of the Netherlands Organisation for Scientific Research (NWO). This work has been 
carried out within the framework of the EUROfusion Consortium and has received funding from the Euratom research and training programme 2014-2018 under grant agreement No 633053. The views and opinions expressed herein do not necessarily reflect those of the European Commission.

\section{References}

1. A. Loarte, Plasma Phys. Control. Fusion, 43, no. 6, pp. R183-R224, (2001)

2. D. Maisonnier, D. Campbell, I. Cook, L. Di Pace, L. Giancarli, J. Hayward, A. Li Puma, M. Medrano, P. Norajitra,M. Roccella, P. Sardain, M.Q. Tran and D. Ward, Nucl. Fusion, 47, 1524 (2007)

3. M. Wischmeier, Journal of Nuclear Materials, 463, 22-29, (2015)

4. S.I. Krasheninnikov, A.S. Kukushkin, and A.A. Pshenov, Physiscs of Plasmas, 23, 055602 (2016)

5. H.J.N van Eck, A.W. Kleyn, A. Lof, H.J. van der Meiden, G.J. van Rooij, J. Scholten and P.A. Zeijlmans van Emmichoven, Applied Physics Letters, 101(22):224107 (2012)

6. G. De Temmerman', M.A. van den Berg, J. Scholten, A. Lof, H.J. van der Meiden, H.J.N. van Eck, T.W. Morgan, T.M. de Kruijf, P.A. Zeijlmans van Emmichoven, J.J. Zielinski, Fusion Engineering and Design, 88, Issues 6-8, 483 (2013)

7. G.J. van Rooij, V.P. Veremiyenko, W.J. Goedheer, B. de Groot, A.W. Kleyn, P.H.M. Smeets, T.W. Versloot, D.G. Whyte, R. Engeln, D.C. Schram and N.J. Lopes Cardozo, Appl. Phys. Lett. 90, 121501 (2007).

8. G. De Temmerman, J.J. Zielinski, S. Van Diepen, L. Marot, and M. Price, Nuclear Fusion, 51(7) (2011)

9. T.W. Morgan, T.M. de Kruif, H.J. van der Meiden, M.A. van den Berg, J. Scholten, W. Melissen, B.J.M. Krijger, S. Bardin, G. De Temmerman, Plasma Phys. Control. Fusion, 56 (9), 095004, (2014) 
10. H.J. van der Meiden, R.S. Al, C.J. Barth, A.J.H. Donné, R. Engeln, W.J. Goedheer, B. de Groot, A.W. Kleyn, W.R. Koppers, O. Kruijt, N.J. Lopes Cardozo, M.J. van de Pol, P.R. Prins, D.C. Schram, A.E. Shumack, P.H.M. Smeets, W.A.J. Vijvers, J. Westerhout, G.M. Wright, and G.J. van Rooij,, Rev. Sci. Instrum.79 (1), 013505-1/8 (2008)

11. H.J. van der Meiden, A.R. Lof, M.A. van den Berg, S. Brons, A.J.H. Donné, H.J.N. van Eck, P.M.J. Koelman, W.R. Koppers, O.G. Kruijt, N.N. Naumenko, T. Oyevaar, P.R. Prins, J. Rapp, J. Scholten, D.C. Schram, P.H.M. Smeets, G. van der Star, S.N. Tugarinov and P.A. Zeijlmans van Emmichoven, Rev. Sci. Instrum.83, 123505 (2012)

12. E.E. Salpeter, Phys. Rev. 120, 1528 (1960)

13. D.E. Evans and J. Katzenstein, Laser light scattering in laboratory plasmas, Rep. Prog. Phys., 32, 207 (1969)

14. P.C. Stangeby, The Plasma Boundery of Magnetic Fusion Devices, IOP Published, (2000)

15. A.E. Shumack, V.P. Veremiyenko, D.C. Schram, H.J. de Blank, W.J. Goedheer, H.J. van der Meiden, W.A.J. Vijvers, J. Westerhout, N.J. Lopes Cardozo and G.J. van Rooij, Phys. Rev. E, 78 (4), 046405 (2008)

16. H.J. van der Meiden, Plasma Phys. Control. Fusion, 52, 045009, (2010)

17. M. Arnaud and R. Rothenflug, Astrophys. Suppl. Ser., 60, 425 (1985)

18. B.D. Fried and S.D. Conte, The Plasma Dispersion Function, Academic Press, New York (1961).

19. T. Wrubel, Stark-breiten isolierter linien in einem mittels ortsaufgelöster Thomson-Streuung diagnostizierten plasma, Ph.D. thesis, Bochum (1999)

20. D.E. Evans, Plasma Phys. 12, 573 (1970)

21. I. Olivares and H.-J. Kunze, J. Appl. Phys., 99, 123305 (2006)

22. H.-J. Kunze, Plasma diagnostics, Lect. Notes Phys. 670, 351, Springer-Verslag Berlin Heidelberg (2005) 
23. E.R. Kieft, J.J.A.M. van der Mullen, G.M.W. Kroesen, V. Banine, and K.N. Koshelev, Phys. Rev E 70, $056413(2004)$

24. H.F. Döbele and K. Gindele, Applied Optics, 16 (9), 2403 (1977)

25. C.J. Barth, C.C. Chu and A.J.H. Donné, Rev. Sci. Instrum, 66, 501 (1995)

26. A.J.H. Donné, A.E. Costley, R. Barnsley, H. Bindslev, R. Boivin,G. Conway, R. Fisher, R. Giannella, H. Hartfuss, M.G. von Hellermann, E. Hodgson, L.C. Ingesson, K. Itami, D. Johnson, Y. Kawano, T. Kondoh, A. Krasilnikov, Y. Kusama, A. Litnovsky, P. Lotte, P. Nielsen, T. Nishitani, F. Orsitto, B.J. Peterson, G. Razdobarin, J. Sanchez, M. Sasao,T. Sugie, G. Vayakis, V. Voitsenya, K. Vukolov, C. Walker, K. Young and the ITPA Topical Group on Diagnostics, Nucl. Fusion 47, S337 (2007)

27. A.E. Shumack, The influence of electric fields and neutral particles on the plasma sheath at ITER divertor conditions, Ph.D. thesis, Eindhoven University of Technology (2011) 